\title{
Effects of Random Density Fluctuations on Matter-Enhanced Neutrino Flavor Transitions in Supernovae and Implications for Supernova Dynamics and Nucleosynthesis
}

\author{
F. N. Loreti, Y.-Z. Qian, \\ G. M. Fuller, and A. B. Balantekin ${ }^{\dagger}$ \\ Institute for Nuclear Theory, \\ University of Washington, Box 351550, Seattle, WA 98195
}

\begin{abstract}
We calculate the effects of random density fluctuations on twoneutrino flavor transformations $\left(\nu_{\tau(\mu)} \rightleftharpoons \nu_{e}\right)$ in the post-core-bounce supernova environment. In particular, we follow numerically the flavor evolution of neutrino states propagating through a stochastic field of density fluctuations. We examine the approach to neutrino flavor depolarization, and study the effects of this phenomenon in both the early shock reheating epoch and the later $r$-process nucleosynthesis epoch. Our results suggest that significant fluctuation-induced neutrino flavor depolarization effects occur in these environments only when the zero-order (without density fluctautions) evolution of the neutrino states includes adiabatic propagation through resonances (mass level crossings). In the shock reheating epoch, depolarization effects from fluctuations with amplitudes larger than $0.05 \%$ of the local matter density can cause an increase in the heating rate of the
\end{abstract}

*Permanent address: Department of Physics, University of California, San Diego, La Jolla, CA 92093-0319.

${ }^{\dagger}$ Permanent address: Department of Physics, University of Wisconsin, Madison, WI 53706. 
material behind the shock compared to the case with no neutrino flavor transformation - but this corresponds to a significant decrease in this quantity relative to the case with adiabatic neutrino transformation. If $r$-process nucleosynthesis is to occur during the late stages of supernova evolution, then the requirement of neutron-rich conditions excludes a region of neutrino mass-squared difference and vacuum mixing angle $\left(\delta m^{2}, \sin ^{2} 2 \theta\right)$ parameter space for neutrino flavor transformation. We find that in the presence of stochastic fluctuations, this excluded region is not significantly altered even for random fluctuations with an amplitude of $1 \%$ of the local matter density. 


\section{INTRODUCTION}

In this paper we investigate matter-enhanced neutrino flavor transformations, the MSW (Mikheyev-Smirnov-Wolfenstein) effect [1,2], for the case of a matter density consisting of a smooth component and a fluctuating part modeled by Gaussian colored noise. We will consider the effects of fluctuation-induced neutrino flavor decoherence during two post-core-bounce epochs of supernova evolution: (1) the shock reheating epoch at TPB (time post bounce) $<1$ s; and $(2)$ the hot bubble $r$-process nucleosynthesis epoch at TPB $\approx 3-16 \mathrm{~s}$.

The neutrino heating of the stalled shock has been examined by Fuller et al. [3] and Wilson and Mayle [4] including neutrino flavor-mixing effects. Flavor mixing between a light $\nu_{e}$ and $\nu_{\mu}$ or $\nu_{\tau}$ with a cosmologically significant mass in the range of $10-100 \mathrm{eV}$ can increase the supernova explosion energy by up to $60 \%$. The increase in the explosion energy is a result of the greater temperature of $\nu_{\mu}$ and $\nu_{\tau}$ compared to that of $\nu_{e}$. Because of their charged current interactions with nucleons, $\nu_{e}$ remain in thermal equilibrium with the matter to lower densities and temperatures than $\nu_{\mu}$ and $\nu_{\tau}$. Thus, although the neutrinos have approximately the same luminosities, flavor transformation between $\nu_{\mu}$ or $\nu_{\tau}$ and $\nu_{e}$ on their way to the stalled shock after thermal decoupling will increase the average energy of $\nu_{e}$, resulting in more heat liberated behind the shock than the case without neutrino flavor transformation.

This increase in the average energy of $\nu_{e}$ by the MSW transformation also affects the possible $r$-process nucleosynthesis of heavy elements in supernovae [5]. In the absence of flavor transitions, $\bar{\nu}_{e}$ have a higher temperature than $\nu_{e}$. This is due to the fact that the neutron-rich surface of the proto neutron star presents a greater opacity to $\nu_{e}$ than $\bar{\nu}_{e}$. Therefore, $\bar{\nu}_{e}$ decouple deeper in the proto neutron star than $\nu_{e}$, and hence, are hotter. Due to their lack of charged current interactions (at these energies) with nucleons, $\nu_{\mu}$ and $\nu_{\tau}$ are even hotter than $\bar{\nu}_{e}$. As pointed out by Qian et al. [5], an MSW transition can result in a proton production rate which is greater than the neutron production rate at the proposed $r$-process site. By requiring neutron-rich conditions at the $r$-process site, Qian et al. were able to put limits on the vacuum mass-squared difference and the vacuum mixing angle between $\nu_{e}$ and $\nu_{\mu}$ or $\nu_{\tau}$. Since $\nu_{\mu}$ and $\nu_{\tau}$ have identical energy spectra, we will hereafter consider only MSW transitions between $\nu_{e}$ and $\nu_{\tau}$ for convenience, although all our results could equally well apply to MSW transitions between $\nu_{e}$ and 
$\nu_{\mu}$.

A general, semi-quantitative approach to neutrino oscillations in inhomogeneous matter was developed in Ref. [6]. Studies of matter density fluctuations which are not random, but harmonic $[7,8]$, or occur as a jumplike change in the solar density [9], are available in the literature. Inhomogeneities in the velocity field of material can mimic the effects of density inhomogeneities on neutrino flavor oscillations [8]. Velocity inhomogeneity effects become important if the characteristic velocity is near the speed of light [8]. Noisy mixing of matter could also mimic a fluctuating matter density.

A priori, fluctuations in the matter density may be well approximated by random noise (which averages to zero) added to the average value of the density. In Ref. [10], a differential equation for the averaged survival probability was derived for the case in which the random noise was taken to be a delta-correlated Gaussian distribution. It was also shown that if the correlation length of the matter density fluctuations was small compared to the neutrino oscillation length at resonance, one obtained the same result as for the case of a delta-correlated Gaussian. Here, we consider the more realistic case of colored noise [11]. We find that the random fluctuations have the largest effect on the flavor transition when the correlation length is on the order of the neutrino oscillation length at resonance, and that the fluctuations, on average, have the effect of suppressing the flavor transition. This result is in agreement with more qualitative arguments regarding flavor transition in an inhomogeneous distribution of matter [6].

Suppressing the degree of flavor transition has the effect of lowering the average energy of $\nu_{e}$ compared with the average energy they would have had for an MSW transition in the absence of the noise. We will show that neutrino flavor decoherence caused by random fluctuations has little effect on the part of the neutrino mixing parameter space $\left(\delta m^{2}, \sin ^{2} 2 \theta\right)$ previously excluded by considerations of MSW neutrino flavor transformation in the hot bubble $r$-process region of the supernova environment. However, we find that neutrino flavor decoherence can have significant effects on the neutrino heating rate and the electron fraction during the shock reheating epoch.

In Sec. II, we develop the equations for two-neutrino flavor conversion in the presence of multiplicative colored noise. We present a differential equation for the averaged transition probability, which is valid when the correlation length is small compared to the width of the resonance region. 
Section III presents our results for the effects of random fluctuations on neutrino heating of the shock and $r$-process nucleosynthesis in the neutrinoheated supernova ejecta. In Sec. IV, we discuss our results and present our conclusions.

\section{NEUTRINO FLAVOR EVOLUTION IN THE PRESENCE OF COLORED NOISE}

In this section, we discuss how an initially pure neutrino flavor state propagating through a stochastic field of density fluctuations can evolve into a mixed ensemble of neutrino flavors. We call this process neutrino flavor decoherence (or flavor depolarization). Here we consider two-neutrino mixing and we assume that there is the usual unitary transformation between flavor eigenstates (e.g., $\left|\nu_{e}\right\rangle$ and $\left.\left|\nu_{\tau}\right\rangle\right)$ and mass eigenstates $\left(\left|\nu_{1}\right\rangle\right.$ and $\left.\left|\nu_{2}\right\rangle\right)$ :

$$
\begin{gathered}
\left|\nu_{e}\right\rangle=\cos \theta\left|\nu_{1}\right\rangle+\sin \theta\left|\nu_{2}\right\rangle \\
\left|\nu_{\tau}\right\rangle=-\sin \theta\left|\nu_{1}\right\rangle+\cos \theta\left|\nu_{2}\right\rangle,
\end{gathered}
$$

where $\theta$ is the vacuum mixing angle. Complete flavor decoherence would occur if a neutrino emitted from the neutrino sphere in an initially pure flavor state, for example $\left|\nu_{e}\right\rangle$, becomes a mixed state of $50 \%\left|\nu_{e}\right\rangle$ and $50 \%$ $\left|\nu_{\tau}\right\rangle$ (both at the original neutrino energy) after propagating through a region where density fluctuations exist.

Our goal in this section is to find which fluctuation characteristics (e.g., root-mean-square amplitude, power spectrum, etc.) would be required to cause significant deviations in neutrino flavor evolution from that predicted by conventional MSW studies with a smooth density run in the post-corebounce supernova environment. We begin with a discussion of the general flavor evolution problem and the way in which fluctuations can be characterized.

The density matrix of two-neutrino flavor evolution obeys the equation

$$
i \frac{d}{d r} \hat{\rho}=[\hat{H}, \hat{\rho}]
$$

where

$$
\hat{\rho} \equiv\left(\begin{array}{c}
a_{e}(r) \\
a_{\tau}(r)
\end{array}\right) \otimes\left(a_{e}^{*}(r), a_{\tau}^{*}(r)\right)
$$


with $a_{e}$ and $a_{\tau}$ the probability amplitudes for the neutrino to be $\nu_{e}$ and $\nu_{\tau}$, respectively, and where the Hamiltonian is given by [1]

$$
\hat{H}=\left(\frac{-\delta m^{2}}{4 E} \cos 2 \theta+\frac{1}{\sqrt{2}} G_{F}\left(N_{e}(r)+N_{e}^{r}(r)\right)\right) \sigma_{z}+\left(\frac{\delta m^{2}}{4 E} \sin 2 \theta\right) \sigma_{x} .
$$

In Eq. (5), $\delta m^{2}$ is the vacuum neutrino mass-squared difference, $E$ is the neutrino energy, $N_{e}$ and $N_{e}^{r}$ are the averaged and randomly fluctuating parts of the electron number density, respectively, and $\sigma_{x}$ and $\sigma_{z}$ are the Pauli matrices.

For colored noise, we take the ensemble averages of the randomly fluctuating part of the electron density to be given by

$$
\begin{gathered}
\left\langle N_{e}^{r}(r)\right\rangle=0, \\
\left\langle N_{e}^{r}(r) N_{e}^{r}\left(r^{\prime}\right)\right\rangle=\beta^{2} N_{e}(r) N_{e}\left(r^{\prime}\right) \exp \left(-\left|r-r^{\prime}\right| / \tau_{c}\right),
\end{gathered}
$$

with the averages of all odd products vanishing and all higher even products given by all possible independent products of two-body correlations (i.e., the fluctuations are Gaussian). For example, if we define $f_{12 \ldots}=$ $\left\langle N_{e}^{r}\left(r_{1}\right) N_{e}^{r}\left(r_{2}\right) \cdots\right\rangle$, the average of a product of four would be $f_{1234}=f_{12} f_{34}+$ $f_{13} f_{24}+f_{14} f_{23}$. In Eq. (7), $\beta$ is the ratio of the root-mean-square fluctuation to the local density, and $\tau_{c}$ is the correlation length.

Clearly, the detailed evolution of the position (time) dependent amplitudes $a_{e}(r)$ and $a_{\tau}(r)$ through regions which include MSW resonances (mass level crossings) is quite complicated in the presence of fluctuations and so necessitates numerical treatment. However, before we proceed to the description of the numerical calculation, it is advantageous to define a few quantities which we shall later employ. First, we introduce the resonance width as

$$
\delta r=2 H \tan 2 \theta,
$$

where the effective weak charge density scale height is $H \equiv\left|d \ln N_{e}(r) / d r\right|^{-1}$ [1]. We define $\Delta \equiv \delta m^{2} / 2 E$, where $\delta m^{2}$ is the difference of the squares of the vacuum neutrino mass eigenvalues. With this notation, resonance (neutrino mass level crossing) occurs at the position where $\Delta \cos 2 \theta=\sqrt{2} G_{F} N_{e}$ is satisfied.

We now proceed to a detailed description of how we follow numerically the time evolution of the neutrino density matrix [i.e., Eq. (3)]. Provided one 
has access to a random number generator which generates Gaussian deviates, Eq. (3) can be integrated by the method of Ref. [11] with the probabilities calculated and averaged. However, such a process can be quite time consuming. For the case in which the correlation length is small compared with the width of the resonance region, a "ladder" approximation can be made allowing one to obtain a differential equation for the averaged probabilities.

In order to obtain this approximation, one can transform $\hat{\rho}$ to the interaction picture, express $\hat{\rho}_{I}$ as an iterative expansion and explicitly perform the averaging to obtain [10]

$$
\left\langle\hat{\rho}_{I}(r)\right\rangle=\frac{1}{2}\left(1+\sigma_{z}\right)+\sum_{n=1}^{\infty}(-1)^{n} \int d R(2 n) F(2 n) \hat{C}(2 n),
$$

where

$$
\begin{gathered}
\int d R(2 n) \equiv \int_{0}^{r} d r_{1} \int_{0}^{r_{1}} d r_{2} \cdots \int_{0}^{r_{2 n-1}} d r_{2 n}, \\
F(2 n) \equiv f_{123 \cdots(2 n-1)(2 n)}, \\
\hat{C}(2 n) \equiv\left[\hat{M}\left(r_{1}\right),\left[\hat{M}\left(r_{2}\right),\left[\cdots,\left[\hat{M}\left(r_{2 n}\right), \sigma_{z}\right] \cdots\right],\right.\right.
\end{gathered}
$$

and $\hat{M}=\left(G_{F} / 2 \sqrt{2}\right) \hat{U}_{0}^{\dagger} \sigma_{z} \hat{U}_{0}$, with $\hat{U}_{0}$ being the propagator of the flavor evolution in the absence of fluctuations. The averaged density matrix is then given by $\langle\hat{\rho}\rangle=U_{0}\left\langle\hat{\rho}_{I}\right\rangle U_{0}^{\dagger}$. From the physical fact that the fluctuations should not affect the evolution far from resonance, one can restrict the integration limits in Eq. (10) to the resonance region. Since the matrix elements of $\hat{C}(2 n)$ are always $\leq 1$, we evaluate the integrals of the terms in $F(2 n)$ and compare the relative size of these terms. When $\tau_{c} \ll \delta r$, the leading term in the integral of $F(2 n)$ is of order $\left(\tau_{c} \delta r\right)^{n}$. This contribution comes from the term in which the subscripts are in the order of the nested integrals [i.e., $\left.\left(f_{12} f_{34} \cdots f_{(2 n-1)(2 n)}\right)\right]$. Terms which are out of this order by $s$ interchanges are of order $(\delta r)^{n-s} \tau_{c}^{n+s}$ when $\tau_{c} \ll \delta r$. Note that because of the absolute value signs in Eq. $(7), f_{i j}=f_{j i}$, so that $f_{13} f_{24} \cdots f_{(2 n-1)(2 n)}$ is out of order by one interchange and $f_{13} f_{45} f_{26} \cdots f_{(2 n-1)(2 n)}$ is out of order by two interchanges. Therefore, the integral of all terms in $F(2 n)$ not in the order of the nested integrals will be of order $\tau_{c} / \delta r$ or smaller than the integral of the term in $F(2 n)$ which is in the order of the nested integrals when $\tau_{c} \ll \delta r$. Therefore, we approximate Eq. (9) by keeping only the largest term in each $F(2 n)$. One 
then obtains an infinite series which is the iterative expansion of

$$
\begin{aligned}
\left\langle\hat{\rho}_{I}(r)\right\rangle & =\frac{1}{2}\left(1+\sigma_{z}\right)-\int_{0}^{r} d r_{1} \int_{0}^{r_{1}} d r_{2} \beta^{2} N_{e}\left(r_{1}\right) N_{e}\left(r_{2}\right) \exp \left[-\left(r_{1}-r_{2}\right) / \tau_{c}\right] \\
& \times\left[\hat{M}\left(r_{1}\right),\left[\hat{M}\left(r_{2}\right),\left\langle\hat{\rho}_{I}\left(r_{2}\right)\right\rangle\right]\right],
\end{aligned}
$$

where we have returned to the full integration limits since the contribution from positions outside the resonance region is small. Equation (13) could also have been obtained by making the assumption $\left\langle N_{e}^{r} N_{e}^{r \prime} \hat{\rho}_{I}\right\rangle \sim\left\langle N_{e}^{r} N_{e}^{r \prime}\right\rangle\left\langle\hat{\rho}_{I}\right\rangle$. Since the maximal reduction in the transition probability occurs for cases in which the correlation length is approximately the neutrino oscillation length at resonance divided by $\pi$ (i.e., $\tau_{c} \sim L_{\text {res }} / \pi=4 E / \delta m^{2} \sin 2 \theta$ ), the above approximation should be valid for adiabatic transitions for which the oscillation length at resonance is much smaller than the width of the resonance region.

As an example of the approximation, we calculate and present the survival probability as a function of the correlation length for a $\nu_{\tau}$ traveling through the supernova at TPB $\approx 3 \mathrm{~s}$ in Fig. 1(a). The rms value of the noise is taken to be $1 \%$ of the local electron number density, and we choose the following parameters: $\delta m^{2}=10^{2} \mathrm{eV}^{2}, \sin ^{2} 2 \theta=10^{-3}$, and $E=33 \mathrm{MeV}$. The correlation length of the random fluctuations varies from $\tau_{c}=(0.25-24) \tau_{c}^{0}$, where $\tau_{c}^{0}=L_{\text {res }} / \pi=\left(\delta m^{2} \sin 2 \theta / 4 E\right)^{-1}=832.6 \mathrm{~cm}$. For these parameters, the neutrino flavor evolution in the absence of noise is highly adiabatic $\left(L_{\mathrm{res}} / \delta r \sim 0.03\right)$. In Fig. 1, the smooth curve is the approximation and the jagged line is the simulation of Eq. (9) utilizing numerical averaging. The survival probability in the absence of fluctuations is not shown in Fig. 1(a), being $\sim 0$. The agreement between the approximation and the simulation is quite good. We also plot in Fig. 1(b) the $\nu_{\tau}$ survival probability as a function of the correlation length for the same parameters as in Fig. 1(a), except with $\sin ^{2} 2 \theta=10^{-5}$ and the corresponding $\tau_{c}^{0}=8326.0 \mathrm{~cm}$. In Fig. 1(b), the horizontal line is the survival probability in the absence of fluctuations. One observes that at small values of the correlation length $\left[\tau_{c} \sim(0-5) \tau_{c}^{0}\right]$, the survival probability from the simulation is larger than that calculated from the approximation, although they are not greatly different.

The reason the approximation works as well as it does is that for both $\tau_{c} \gg L_{\mathrm{res}}$ and $\tau_{c} \ll L_{\mathrm{res}}$, the approximation and the numerically-averaged simulation approach the probability in the absence of fluctuations. As discussed in Ref. [10], the parameter $\gamma=1 / 2 G_{F}^{2}\left\langle\left(N_{e}^{r}\right)^{2}\right\rangle \tau_{c} \delta r$ governs the size of the effect of the fluctuations when $\tau_{c} \ll L_{\text {res }}$. When $\gamma$ is large, the transition 
probability is heavily suppressed, where as for $\gamma \ll 1$, the fluctuations have little effect. Therefore, a small value for $\tau_{c}$ will result in only a small change in the probability. As $\tau_{c}$ goes to infinity,

$$
\begin{aligned}
\lim _{\tau_{c} \rightarrow \infty} F(2 n) & =(2 n-1) ! ! \beta^{2 n} \prod_{i=1}^{2 n} N_{e}\left(r_{i}\right) \\
& =\frac{1}{\sqrt{2 \pi \beta^{2}}} \int_{-\infty}^{\infty} d x \exp \left[-x^{2} /\left(2 \beta^{2}\right)\right] x^{2 n} \prod_{i=1}^{2 n} N_{e}\left(r_{i}\right)
\end{aligned}
$$

and Eq. (9) can be summed and transformed back from the interaction picture to give

$$
\lim _{\tau_{c} \rightarrow \infty}\langle\hat{\rho}(r)\rangle=\frac{1}{\sqrt{2 \pi \beta^{2}}} \int_{-\infty}^{\infty} d x \exp \left[-x^{2} /\left(2 \beta^{2}\right)\right] \hat{\rho}(r, x),
$$

where $\hat{\rho}(r, x)$ is the density matrix calculated using Eq. (3) for the electron density $(1+x) N_{e}(r)$. Equation (15) provides a simple physical picture for the averaged density matrix in the limit of very large correlation lengths: one simply calculates the density matrix for the electron density with a "frozen" fluctuation, $(1+x) N_{e}(r)$, then averages over all such fluctuations with a Gaussian weight. Large correlation lengths imply that fluctuations at many different locations are coupled. Indeed, an averaging such as given in Eq. (15) is common in other physical situations when many coupled channels are present. For example, in multidimensional dissipative quantum tunnelling, barrier transmission probability is given by a similar formula when the number of channels gets very large [12]. Note that to first order in $x$, the change $N_{e}(r) \rightarrow(1+x) N_{e}(r)$ in the functional form of the density will affect the survival probability via a change in the slope of the density at resonance. For slowly changing slopes and small $\beta$, the change in the survival probability should be proportional to $\beta^{2}$, and therefore be quite small.

\section{EFFECTS ON THE $r$-PROCESS AND SHOCK REHEATING}

As demonstrated by Fig. 1(b), even a 1\% fluctuation in the matter density (a value which is probably unrealistically large for the rather quiescent TPB 
$>3 \mathrm{~s}$ epoch) does little in reducing the transition probability (or increasing the survival probability) for non-adiabatic evolution. As shown in Ref. [5], the neutrino-heated supernova ejecta must have a neutron excess $\left(Y_{e}<0.5\right)$ in order for any $r$-process nucleosynthesis to be produced. In Ref. [5], it was shown that the electron fraction $Y_{e}$ is approximately given by

$$
Y_{e} \approx \frac{1}{1+\lambda_{\bar{\nu}_{e} p} / \lambda_{\nu_{e} n}} \approx \frac{1}{1+\left\langle E_{\bar{\nu}_{e}}\right\rangle /\left\langle E_{\nu_{e}}\right\rangle}
$$

where $\lambda_{\bar{\nu}_{e} p}$ and $\lambda_{\nu_{e} n}$ are the reaction rates for

$$
\begin{aligned}
& \bar{\nu}_{e}+p \rightarrow e^{+}+n \\
& \nu_{e}+n \rightarrow e^{-}+p
\end{aligned}
$$

respectively, and $\left\langle E_{\nu_{e}}\right\rangle$ and $\left\langle E_{\bar{\nu}_{e}}\right\rangle$ are the average energy for $\nu_{e}$ and $\bar{\nu}_{e}$, respectively. Since at this epoch the average neutrino energies are $\left\langle E_{\nu_{e}}\right\rangle \approx 11$ $\mathrm{MeV},\left\langle E_{\bar{\nu}_{e}}\right\rangle \approx 16 \mathrm{MeV}$, and $\left\langle E_{\nu_{\tau}}\right\rangle \approx 25 \mathrm{MeV}$, a substantial conversion of $\nu_{\tau}$ into $\nu_{e}$ can decrease the ratio $\left\langle E_{\bar{\nu}_{e}}\right\rangle /\left\langle E_{\nu_{e}}\right\rangle$ below one and thereby make conditions impossible for the $r$-process. The average $\nu_{e}$ energy after an MSW transition is approximately $P\left(\nu_{e} \rightarrow \nu_{e}\right)\left\langle E_{\nu_{e}}\right\rangle+P\left(\nu_{\tau} \rightarrow \nu_{e}\right)\left\langle E_{\nu_{\tau}}\right\rangle$. To obtain neutron-rich conditions, one must have $P\left(\nu_{e} \rightarrow \nu_{e}\right)>64 \%$. It only requires about $30 \%$ efficiency in flavor conversion to drive the neutrino-heated supernova ejecta too proton rich for $r$-process nucleosynthesis. Consider simple estimates for $Y_{e}$ using Eq. (16) for four cases:

(1) No flavor mixing. In this case, we have $Y_{e} \approx 1 /\left(1+\left\langle E_{\bar{\nu}_{e}}\right\rangle /\left\langle E_{\nu_{e}}\right\rangle\right) \approx$ $1 /(1+16 / 11) \approx 0.41$, so the material is neutron rich, in good agreement with detailed supernova model calculations [5].

(2) Full flavor conversion. In this case, we have $\left\langle E_{\nu_{e}}\right\rangle \rightleftharpoons\left\langle E_{\nu_{\tau}}\right\rangle$, so that $Y_{e} \approx 1 /\left(1+\left\langle E_{\bar{\nu}_{e}}\right\rangle /\left\langle E_{\nu_{\tau}}\right\rangle\right) \approx 1 /(1+16 / 25) \approx 0.61$, and the material is very proton rich.

(3) Complete neutrino flavor depolarization. In this case, there is $50 \%$ flavor conversion, so $\left\langle E_{\nu_{e}}\right\rangle \rightarrow\left(\left\langle E_{\nu_{e}}\right\rangle+\left\langle E_{\nu_{\tau}}\right\rangle\right) / 2 \approx 18 \mathrm{MeV}$, which implies that $Y_{e} \approx 1 /\left(1+\left\langle E_{\bar{\nu}_{e}}\right\rangle /\left\langle E_{\nu_{e}}^{\prime}\right\rangle\right) \approx 0.53$, too proton rich for $r$-process nucleosynthesis.

In fact, we must have $Y_{e}<0.5$ to get any $r$-process nucleosynthesis. This is a conservative limit, since a good $r$-process requires $Y_{e} \leq 0.45$. Now consider a fourth case, 
(4) Partial flavor depolarization. For $35 \%$ conversion of $\nu_{\tau}$ into $\nu_{e}$, we have $Y_{e} \approx 0.5$. For $30 \%$ conversion of $\nu_{\tau}$ into $\nu_{e}$, we have $Y_{e} \approx 0.49$, realistically too large to give acceptable $r$-process nucleosynthesis. In fact, to get $Y_{e}<0.45$ we need to demand that there had been less than $15 \%$ flavor conversion.

Consider some condition along the $Y_{e}=0.5$ line in Fig. 2 of Ref. [5]. For example, consider $E_{\nu}=25 \mathrm{MeV}$ and $\delta m^{2} \approx 900 \mathrm{eV}^{2}$, for which the density scale height at resonance is $H \approx 0.5 \mathrm{~km}$. This value of $\delta m^{2}$ corresponds to $\sin ^{2} 2 \theta \approx 4 \times 10^{-6}$ on the $Y_{e}=0.5$ line. In this case, the conversion probability is $P\left(\nu_{\tau} \rightarrow \nu_{e}\right) \approx 1-\exp \left\{-0.04\left(\delta m^{2} / \mathrm{eV}^{2}\right)\left(\mathrm{MeV} / E_{\nu}\right)(H / \mathrm{cm}) \sin ^{2} 2 \theta\right\} \approx$ $25 \%$. The neutrino energies around $25 \mathrm{MeV}$ are the most important in terms of leverage on $Y_{e}$. Note that fluctuation-induced depolarization at a level of $50 \%$ could conceivably produce more conversion than MSW transformation at $E_{\nu}=25 \mathrm{MeV}$ over a considerable part of the region to the right of the $Y_{e}=0.5$ line in Fig. 2 of Ref. [5]. As outlined above, greater than $30 \%$ flavor conversion will always drive the material too proton rich for $r$-process.

One can conclude that the random fluctuations will have a quite minor effect on the neutrino mixing parameters constrained by $r$-process nucleosynthesis because the absolute increase of the survival probability rapidly diminishes with increasing survival probability. To illustrate this, we plot the survival probability as a function of energy for $\nu_{\tau}$ with fluctuations of $1 \%$ and $0.5 \%$ of the local matter density in Figs. 2(a) and 2(b). We choose the parameters of Fig. 1(b) so that the evolution is nonadiabatic, and the neutrino energy is chosen to maximize the differential capture rate [see Eq. (23)]. In Fig. 2, the jagged lines are the solution using Eq. (9), the solid line is the approximate solution, and the dashed line is the survival probability in the absence of fluctuations. One observes that the approximate solution does fairly well in reproducing the simulation. There is a small increase of about 0.08 in the survival probability in Fig. 2(a) where the fluctuations are $1 \%$ of the local density, but only a very small increase is obtained for $0.5 \%$ noise in Fig. 2(b). If one calculates $Y_{e}$ for these parameters in the absence of fluctuations, one obtains $Y_{e}=0.5$ indicating that this point lies on the boundary of the excluded region of the MSW parameter space [5]. The inclusion of noise at the $1 \%$ level would decrease $Y_{e}$ by about $1 \%$. Random fluctuations of $0.5 \%$ as in Fig. 2(b) would give a decrease of about $0.4 \%$. For noise at the $0.1 \%$ level, there will essentially be no change in the excluded region. 
Note that if the neutrino flavor transition is adiabatic, the fluctuations can increase the survival probability from approximately zero to one half. The shock reheating epoch occurs at approximately TPB $\sim 0.15 \mathrm{~s}$, and the relevant scale heights are larger than those at TPB $>3 \mathrm{~s}$, which implies a larger resonance width for comparable values of $\delta m^{2}$ and $\sin ^{2} 2 \theta$. Hence, $\gamma$ may remain $\sim 1$ while $\left\langle\left(N_{e}^{r}\right)^{2}\right\rangle$ is reduced allowing one to obtain a sizable effect for adiabatic transitions as shown in Fig. 1(a) for TPB $\approx 3 \mathrm{~s}$ with a smaller rms value of the noise. In Fig. 3 we show the survival probability as a function of neutrino energy for the values $\delta m^{2}=10^{3} \mathrm{eV}^{2}, \sin ^{2} 2 \theta=10^{-6}$, $\tau_{c}=2.762 \times 10^{3} \mathrm{~cm}$. The rms fluctuations are $0.05 \%$ and $0.1 \%$ of the local density in Figs. 3(a) and 3(b), respectively. In both cases there is a large region where the survival probability is increased from the value of essentially zero in the absence of fluctuations. The approximate solution seems to be better in Fig. 3(b) where the rms value of the fluctuations is larger, but is reasonably close to the numerical simulation in both cases. For the case of an adiabatic transition between the more energetic $\nu_{\tau}$ and less energetic $\nu_{e}$, the heating rate can be increased by $(30-60) \%$ [3]. Therefore, the reduction of the transition probability by random fluctuation effects for an a priori reasonable rms value of the fluctuations [(0.05-0.1)\%] could be important.

We can estimate the decrease in the heating rate due to the fluctuations over that from the case of an adiabatic MSW transition as follows. In the absence of neutrino flavor conversion, the heating rate per nucleon is approximately given by [13]

$$
\dot{\epsilon}_{\nu N} \approx \frac{L_{\nu}}{4 \pi r^{2}} \frac{\int_{0}^{\infty} E_{\nu}^{3} \sigma_{\nu N} d E_{\nu} /\left(\exp \left(E_{\nu} / T_{\nu}\right)+1\right)}{\int_{0}^{\infty} E_{\nu}^{3} d E_{\nu} /\left(\exp \left(E_{\nu} / T_{\nu}\right)+1\right)}=\int_{0}^{\infty} E_{\nu} \sigma_{\nu N} \frac{d \phi\left(E_{\nu}, T_{\nu}\right)}{d E_{\nu}} d E_{\nu}
$$

where

$$
\sigma_{\nu N} \approx 9.6 \times 10^{-44}\left(\frac{E_{e}}{\mathrm{MeV}}\right)^{2} \mathrm{~cm}^{2},
$$

is the absorption cross section, $L_{\nu}$ is the neutrino luminosity (assumed equal for all species), $T_{\nu}$ is the neutrino temperature, $d \phi$ is the differential neutrino flux with respect to neutrino energy, and $E_{e}$ is the energy of the produced electron or positron in Eqs. (17) and (18).

The total heating rate combining contributions from both $\nu_{e}$ and $\bar{\nu}_{e}$ is

$$
\dot{\epsilon}_{\text {tot }}=Y_{n} \dot{\epsilon}_{\nu_{e} n}+Y_{p} \dot{\bar{\nu}}_{\bar{e} p}
$$


where $Y_{n}$ and $Y_{p}$ are the number fractions of free neutrons and protons, respectively, with $Y_{n}+Y_{p} \approx 1$. Of course, $Y_{n}$ and $Y_{p}$ will be set by the same weak interactions responsible for heating the shock. In the region where neutrino heating dominates, we can assume

$$
\frac{Y_{n}}{Y_{p}} \approx \frac{\lambda_{\bar{\nu}_{e} p}}{\lambda_{\nu_{e} n}}
$$

where $\lambda_{\nu N}$ is the neutrino capture rate on free nucleons and is given by

$$
\lambda_{\nu N} \approx \int_{0}^{\infty} \sigma_{\nu N} \frac{d \phi\left(E_{\nu}, T_{\nu}\right)}{d E_{\nu}} d E_{\nu}
$$

An MSW transition between $\nu_{e}$ and $\nu_{\tau}$ will increase the heating rate by a factor of

$$
\frac{\dot{\epsilon}_{t o t}^{\prime}}{\dot{\epsilon}_{t o t}}=\frac{Y_{n}^{\prime} \dot{\epsilon}_{\nu_{e} n}^{\prime}+Y_{p}^{\prime} \dot{\epsilon}_{\bar{\nu}_{e} p}}{Y_{n} \dot{\epsilon}_{\nu_{e} n}+Y_{p} \dot{\epsilon}_{\bar{\nu}_{e} p}},
$$

where $\dot{\epsilon}_{\nu_{e} n}^{\prime}$ is given by

$$
\begin{aligned}
\dot{\epsilon}_{\nu_{e} n}^{\prime} & =\int_{0}^{\infty} P\left(E_{\nu}, \tau_{c}, \beta\right) E_{\nu} \sigma_{\nu N} \frac{d \phi\left(E_{\nu}, T_{\nu_{e}}\right)}{d E_{\nu}} d E_{\nu} \\
& +\int_{0}^{\infty}\left[1-P\left(E_{\nu}, \tau_{c}, \beta\right)\right] E_{\nu} \sigma_{\nu N} \frac{d \phi\left(E_{\nu}, T_{\nu_{\tau}}\right)}{d E_{\nu}} d E_{\nu} .
\end{aligned}
$$

We take $Y_{n}^{\prime}$ and $Y_{p}^{\prime}$ to be given by Eq. (22) with

$$
\begin{aligned}
\lambda_{\nu_{e} n}^{\prime} & =\int_{0}^{\infty} P\left(E_{\nu}, \tau_{c}, \beta\right) \sigma_{\nu N} \frac{d \phi\left(E_{\nu}, T_{\nu_{e}}\right)}{d E_{\nu}} d E_{\nu} \\
& +\int_{0}^{\infty}\left[1-P\left(E_{\nu}, \tau_{c}, \beta\right)\right] \sigma_{\nu N} \frac{d \phi\left(E_{\nu}, T_{\nu_{\tau}}\right)}{d E_{\nu}} d E_{\nu}
\end{aligned}
$$

In the above equations, $P\left(E_{\nu}, \tau_{c}, \beta\right)$ is the survival probability of $\nu_{\tau}$ (or $\nu_{e}$ ).

We have calculated Eq. (24) for the cases of random fluctuations with an rms value of $0.05 \%$ and $0.1 \%$ of the local matter density, and for the case without fluctuations. We present the results in Table I. For each set of values for $\left(\delta m^{2}, \sin ^{2} 2 \theta\right)$, we have taken $\tau_{c}=\left(\delta m^{2} \sin 2 \theta / 4 E_{\text {peak }}\right)^{-1}$, where $E_{\text {peak }}=$ $5 T_{\nu_{\tau}}$, the energy for which the integrand is approximately maximized. We have taken the temperatures to be $T_{\nu_{e}}=T_{\bar{\nu}_{e}} \approx 5 \mathrm{MeV}$ and $T_{\nu_{\tau}} \approx 7 \mathrm{MeV}$. The values in Table I were calculated using the approximation in Eq. (13) rather 
than the "exact" numerical simulation, since we are interested in estimating the size of the difference in neutrino flavor conversion efficiency for the cases of smooth and noisy density distributions, not on a particular model of the fluctuations which may or may not obey Eqs. (6) and (7). From Table I, one sees that the noise can reduce the heating rate by up to $45 \%$ from the adiabatic MSW case.

\section{DISCUSSION AND CONCLUSIONS}

To our knowledge, there is no consensus on the size of possible matter density fluctuations in post-core-bounce supernovae. Although we have taken the rms size of the fluctuations to be a constant fraction of the local matter density, it may very well increase or decrease with decreasing density, or the size could depend on the distance from the shock. Similarly, the correlation lengths we have used were chosen to give the maximal reduction in the MSW transition probability and were independent of the density and the distance from the shock. If, for instance, the correlation length increased with decreasing density, the effect of the noise in reducing the MSW transition probability could be enhanced since for fixed $\delta m^{2}$ and $\sin ^{2} 2 \theta$ the correlation length of maximal effect $\left(\tau_{c} \sim L_{\text {res }}\right)$ varies linearly with the neutrino energy. Our intention in this paper is to establish the maximal effect density fluctuations could have if they can be well approximated by random noise added to the average density.

For an rms fluctuation value of $1 \%$, the addition of the noise will have a slight effect on the $r$-process nucleosynthesis as compared with the MSW effect in the absence of noise. This can be traced to the fact that demanding neutron-rich conditions at the site of the $r$-process eliminates all but the nonadiabatic region of the neutrino mixing parameter space. The effect of the noise is large in the adiabatic region only and becomes increasingly negligible as the neutrino flavor transition becomes nonadiabatic. A $1 \%$ fluctuation will decrease $Y_{e}$ from 0.5 to $\sim 0.495$ for points in the MSW parameter space on the boundary of the excluded region [5]. However, we consider this rms fluctuation value to be actually too large for the relatively quiesent TPB $>3$ s epoch. For an order of magnitude smaller fluctuation which may be more reasonable, the effect of the noise is totally ignorable. In other words, noise at the $0.1 \%$ level will not alter the region of the neutrino mixing parameter 
space excluded in Ref. [5] by the MSW effect.

Noise with an rms amplitude of $0.05 \%$ of the averaged local matter density can lead to significant neutrino flavor decoherence during the shock reheating epoch at TPB $\sim 0.15 \mathrm{~s}$. In turn, this would lead to a dimunition of the adiabatic MSW-induced increase in the supernova explosion energy from $\sim$ $(30-60) \%$ to $\sim 20 \%$. The physics of supernova explosion process is quite complicated and depends on many factors. In our opinion, if the heating of the stalled shock by an MSW transition is determined to be a necessary component for a successful explosion, then a more detailed analysis of the effects of random matter density fluctuations on the MSW transition would be warranted.

We wish to thank Ray Sawyer for bringing this problem to our attention and providing many valuable insights. We also want to thank Wick Haxton and Lincoln Wolfenstein for very useful discussions. This work was supported by the Department of Energy under Grant No. DE-FG06-90ER40561 at the Institute for Nuclear Theory, by NSF Grant No. PHY-9503384 at UCSD, by NSF Grant No. PHY-9314131 and by the Wisconsin Alumni Research Foundation at UW. 


\section{References}

[1] L. Wolfenstein, Phys. Rev. D 17, 2369 (1978); 20, 2634 (1979). S. P. Mikheyev and A. Yu. Smirnov, Nuovo Cim. 9C, 17 (1986); Sov. J. Nucl. Phys. 42, 913 (1986); H. A. Bethe, Phys. Rev. Lett. 56, 1305 (1986).

[2] W. C. Haxton, Phys. Rev. Lett. 57, 1271 (1986); S. J. Parke, ibid. 57, 1275 (1986); S. P. Rosen and J. M. Gelb, Phys. Rev. D 34, 969 (1986); A. B. Balantekin, S. H. Fricke, and P. J. Hatchell, Phys. Rev. D 38, 935 (1988); J. N. Bahcall and H. A. Bethe, Phys. Rev. Lett. 65, 2233 (1990).

[3] G. M. Fuller, R. W. Mayle, B. S. Meyer and, J. R. Wilson, Astrophys. J. 389, 517 (1992); G. M. Fuller, Phys. Rep. 227, 149 (1993).

[4] J. R. Wilson and R. W. Mayle, private communication.

[5] Y.-Z. Qian, G. M. Fuller, G. J. Mathews, R. W. Mayle, J. R. Wilson, S. E. Woosley, Phys. Rev. Lett. 71, 1965 (1993).

[6] R. F. Sawyer, Phys. Rev. D 42, 3908 (1990).

[7] A. Schafer and S. E. Koonin, Phys. Lett. B 185, 417 (1987).

[8] W. C. Haxton and W-M. Zhang, Phys. Rev. D 43, 2484 (1991).

[9] P. I. Krastev and A. Yu. Smirnov, Mod. Phys. Lett. A 6, 1001 (1991).

[10] F. N. Loreti and A. B. Balantekin, Phys. Rev. D 50, 4762, (1994).

[11] R. Mannella and V. Palleschi, Phys. Rev. A 40, 3381 (1989).

[12] A. B. Balantekin and N. Takigawa, Ann. Phys. (New York) 160, 441 (1985).

[13] Y.-Z. Qian, Ph.D. thesis, University of California, San Diego (1993). 
Table I. Ratios of the heating rates in the presence of MSW transitions with no fluctuations $(\beta=0), 0.05 \%$ fluctuations $\left(\beta=5 \times 10^{-4}\right)$, and $0.1 \%$ fluctuations $\left(\beta=10^{-3}\right)$ to the heating rate in the absence of flavor transitions. The neutrino temperatures are $T_{\nu_{e}}=T_{\bar{\nu}_{e}}=5 \mathrm{MeV}$ and $T_{\nu_{\tau}}=7 \mathrm{MeV}$.

\begin{tabular}{ccccc}
\hline \hline$\delta m^{2}\left(\mathrm{eV}^{2}\right)$ & $\sin ^{2} 2 \theta$ & $\beta=0$ & $\beta=5 \times 10^{-4}$ & $\beta=10^{-3}$ \\
\hline $5 \times 10^{2}$ & $2 \times 10^{-8}$ & 1.05 & 1.05 & 1.05 \\
$5 \times 10^{2}$ & $10^{-7}$ & 1.16 & 1.14 & 1.12 \\
$5 \times 10^{2}$ & $10^{-6}$ & 1.31 & 1.23 & 1.18 \\
$10^{3}$ & $2 \times 10^{-8}$ & 1.07 & 1.07 & 1.06 \\
$10^{3}$ & $10^{-7}$ & 1.23 & 1.19 & 1.17 \\
$10^{3}$ & $10^{-6}$ & 1.35 & 1.23 & 1.19 \\
$8 \times 10^{3}$ & $2 \times 10^{-8}$ & 1.12 & 1.11 & 1.11 \\
$8 \times 10^{3}$ & $10^{-7}$ & 1.31 & 1.19 & 1.19 \\
$8 \times 10^{3}$ & $10^{-6}$ & 1.35 & 1.19 & 1.19 \\
\hline \hline
\end{tabular}




\section{Figure Captions}

Figure 1. Survival probability $P\left(\nu_{\tau} \rightarrow \nu_{\tau}\right)$ is given as a function of the correlation length, $\tau_{c}$ for the numerical simulation by the method of Ref. [11] and by the approximation in Eq. (9). The parameters chosen were $\delta m^{2}=10^{2}$ $\mathrm{eV}^{2}, \sin ^{2} 2 \theta=10^{-3}, E=33 \mathrm{MeV}$, and $\tau_{c}^{0}=L_{\mathrm{res}} / \pi=\left(\delta m^{2} \sin 2 \theta / 4 E\right)^{-1}=$ $832.6 \mathrm{~cm}$ for (a). The values $\delta m^{2}=10^{2} \mathrm{eV}^{2}, \sin ^{2} 2 \theta=10^{-5}, E=33 \mathrm{MeV}$, and $\tau_{c}^{0}=8326 \mathrm{~cm}$ were chosen for $(\mathrm{b})$. The rms value of the fluctuations was $1 \%$ of the local density in both (a) and (b). The matter density profile at TPB $\approx 3$ s was used [5] for both (a) and (b). The horizontal line in (b) is the survival probability in the absence of fluctuations.

Figure 2. Survival probability $P\left(\nu_{\tau} \rightarrow \nu_{\tau}\right)$ is given as a function of energy for the numerical simulation by the method of Ref. [11] and by the approximation in Eq. (9). The rms value of the fluctuations is taken to be $1 \%$ in (a) and $0.5 \%$ in (b). In both (a) and (b) the survival probability in the absence of the fluctuations is shown by the dashed line. The parameters chosen were $\delta m^{2}=10^{2} \mathrm{eV}^{2}, \sin ^{2} 2 \theta=10^{-5}, \tau_{c}=8326 \mathrm{~cm}$. The matter density profile at $\mathrm{TPB} \approx 3 \mathrm{~s}$ was used [5].

Figure 3. Survival probability $P\left(\nu_{\tau} \rightarrow \nu_{\tau}\right)$ is given as a function of energy for the numerical simulation by the method of Ref. [11] and by the approximation in Eq. (9). The rms value of the fluctuations is $0.05 \%$ in (a) and $0.1 \%$ in (b). In both (a) and (b) the survival probability in the absence of the fluctuations is essentially zero. The parameters chosen were $\Delta m^{2}=10^{3} \mathrm{eV}^{2}$,

$\sin ^{2} 2 \theta=10^{-6}, \tau_{c}=2792.4 \mathrm{~cm}$. The matter density profile at $\mathrm{TPB} \approx 0.15 \mathrm{~s}$ was used [4] and the density profile was cut off at a radius corresponding to the position of the stalled shock. 


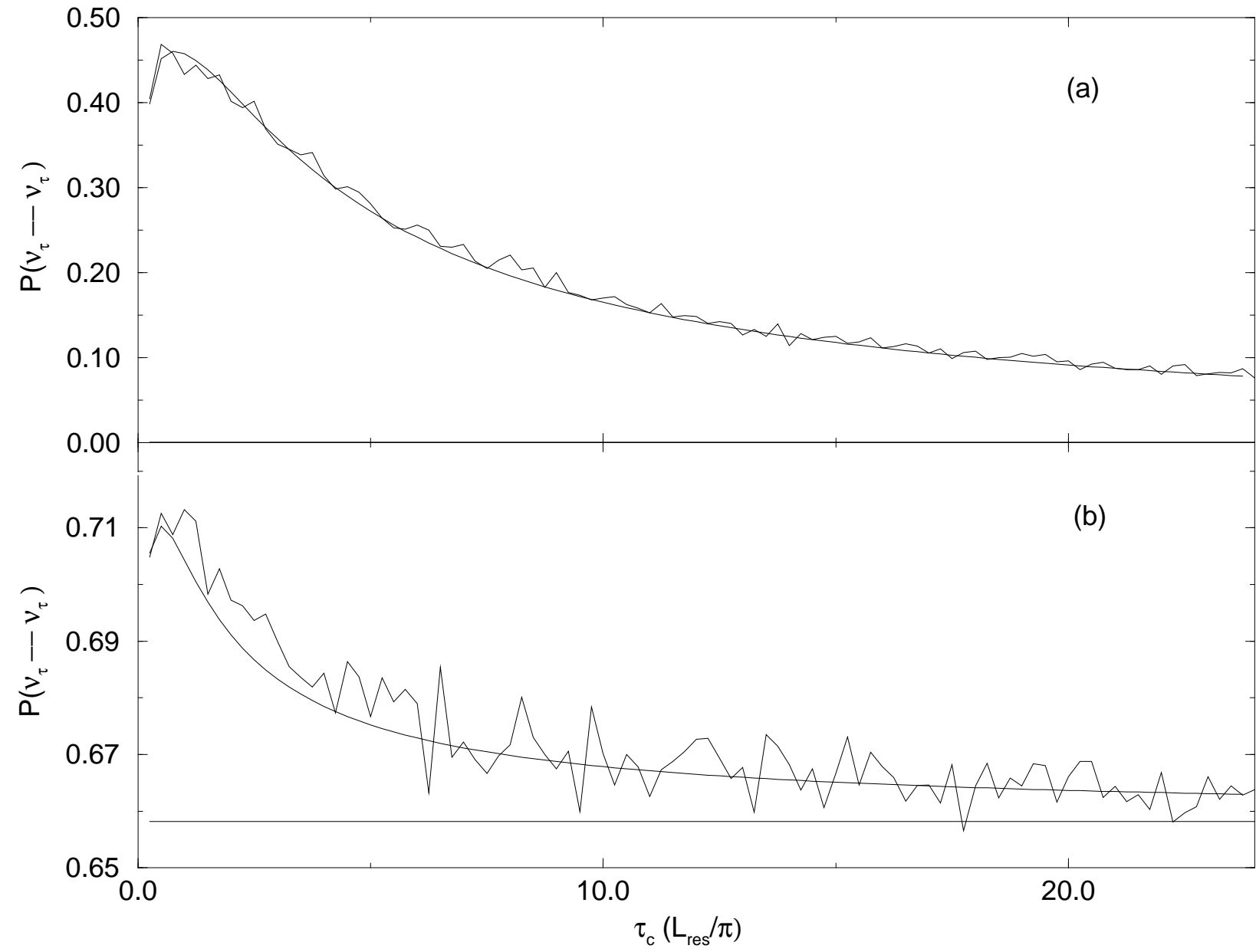




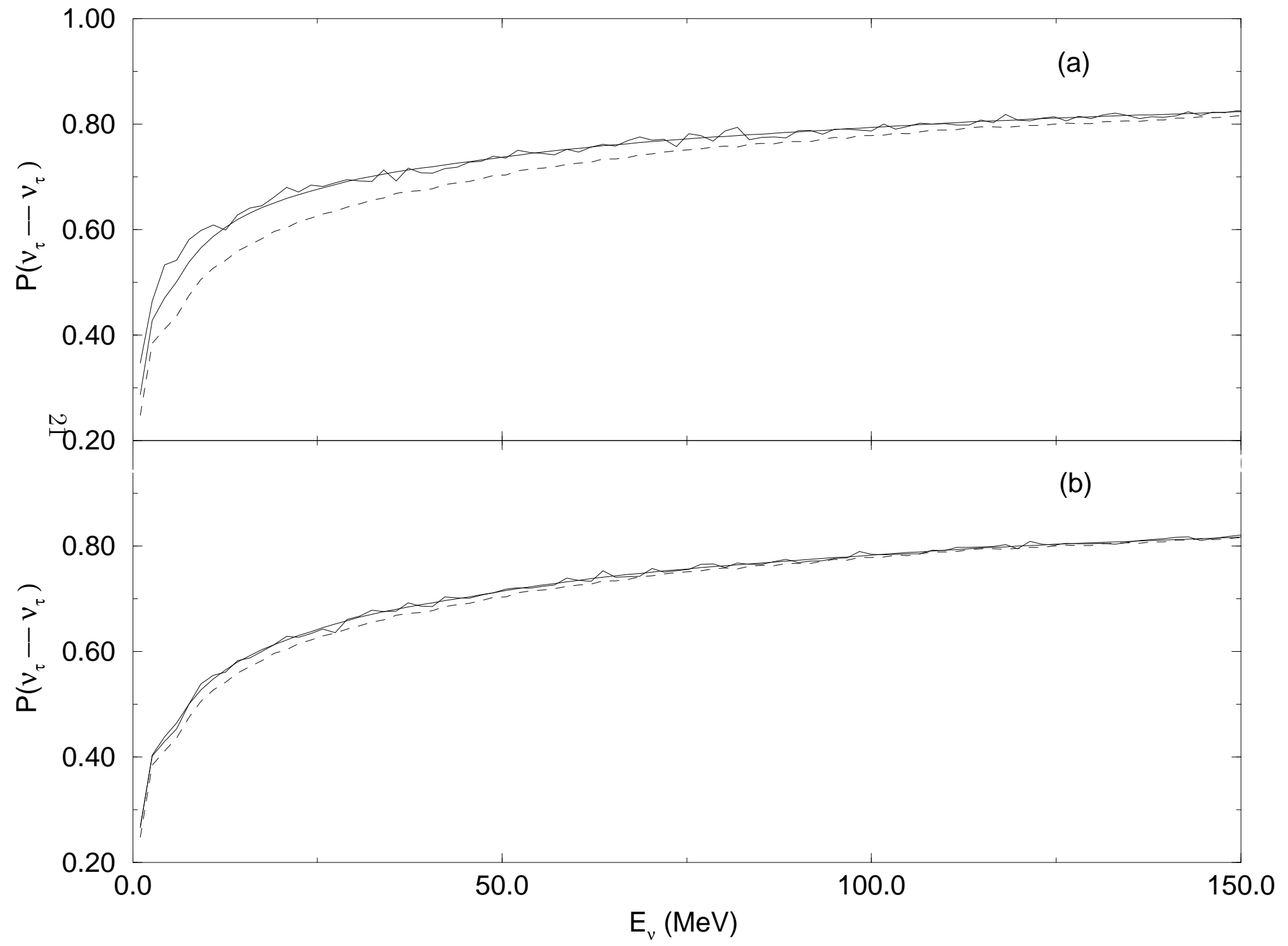




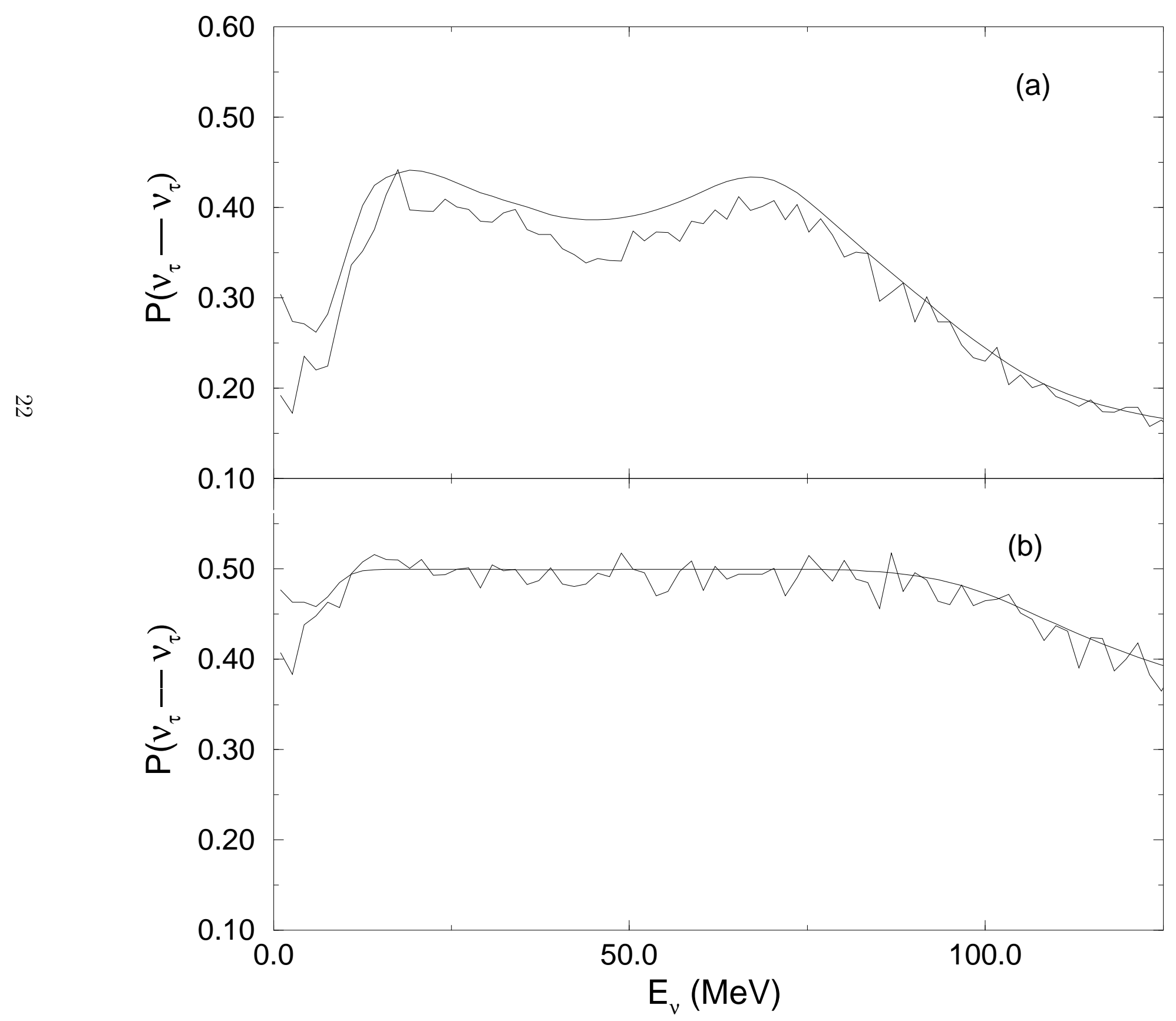

